\title{
Increased cryo-survival rate in ejaculated human sperm from infertile men following pre-freeze in vitro myo-inositol supplementation
}

\author{
Ramadan Saleh ${ }^{1,2}$, Hanan Assaf', Wafaa M. Abd El Maged' ${ }^{1}$, Mohamed Elsuity' ${ }^{1}$, Mohamed Fawzy ${ }^{2}$ \\ 'Department of Dermatology, Venereology and Andrology, Faculty of Medicine, Sohag University, Sohag; ${ }^{2}$ Ajyal IVF Center, Ajyal Hospital, Sohag, Egypt
}

Objective: To investigate the effects of in vitro myo-inositol (Myo-Ins) supplementation of cryopreserved human semen on the cryo-survival rate (CSR).

Methods: Semen samples were obtained from 41 infertile men. Following routine semen analysis, each sample was divided into two equal aliquots ( $0.5 \mathrm{~mL}$ each). One aliquot was treated with $1 \mathrm{mg}$ of Myo-Ins dissolved in $10 \mu \mathrm{L}$ of sperm preparation medium. The second aliquot was treated with $10 \mu \mathrm{L}$ of the same medium (control). Both aliquots were incubated for 20 minutes prior to freezing to slow the freezing process. The frozen samples were examined for post-thaw percentages of total motility (TM), progressive motility (PM), and the CSR, defined as the percentage of post-thaw TM divided by the percentage of pre-freeze TM and multiplied in 100 . The results were expressed as median and interquartile range (25th and 75 th percentiles).

Results: The pre-freeze TM (50\% [30\%-50\%]) and PM (35\% [20\%-35\%]) were significantly higher than the post-thaw TM and PM in the MyoIns group (15\% [10\%-35\%] and 10\% [5\%-20\%]; $p<0.001$ and $p<0.001$, respectively) and the control group (10\% [6\%-30\%] and 5\% [3\%$15 \%] ; p<0.001$ and $p<0.001$, respectively). The CSR of the 41 semen aliquots supplemented with Myo-Ins ( $40 \%$ [25\%-70\%]) was significantly higher than that of the control samples (30\% [13\%-58\%], $p=0.041)$. The CSR of the 26 abnormal semen samples that were supplemented with Myo-Ins (38\% [20\%-50\%]) was significantly higher than that of the control samples (23\% [12\%-30\%], $p=0.031$ ).

Conclusion: In vitro Myo-Ins supplementation of ejaculated human sperm from infertile men resulted in a significant increase in the CSR in samples with abnormal pre-freeze sperm parameters.

Keywords: Cryo-survival rate; Human sperm; Infertile men; Myo-inositol; Sperm cryopreservation

\section{Introduction}

Sperm cryopreservation is the only available method of preserving male reproductive potential, and has been used widely since the 1970s to treat couples with infertility [1]. Sperm banking has become an important part of any assisted reproductive technology (ART)

Received: May 23, 2018 · Revised: Oct 16, 2018 · Accepted: Oct 23, 2018 Corresponding author: Ramadan Saleh

Department of Dermatology, Venereology and Andrology, Faculty of Medicine, Sohag University, Sohag 82524, Egypt

Tel:+20-0932304772 Fax:+20-0932304773 E-mail:salehr2010@yahoo.com

This is an Open Access article distributed under the terms of the Creative Commons Attribution Non-Commercial License (http://creativecommons.org/licenses/by-nc/4.0/) which permits unrestricted non-commercial use, distribution, and reproduction in any medium, provided the original work is properly cited. program, and is essential for preserving fertility in male patients with cancer and some autoimmune diseases [2]. There is growing interest in fertility preservation of patients with cancer or systematic diseases since survival rates are increasing due to new therapeutic options [3]. However, the results of sperm cryopreservation are limited by a decrease in the number of viable spermatozoa, mainly due to the detrimental effects of the cryopreservation process on the plasma membrane of sperm $[4,5]$. The damaging effects of freezing and thawing on the plasma membrane of sperm may reduce the fertilizing capacity of spermatozoa [6]. Sperm freezing has also been associated with impaired sperm motility [7], alterations of sperm morphology [8], and damage to the acrosome [9]. In addition, the nuclear DNA of sperm in infertile men was found to be significantly damaged by the 
freezing-thawing process [10]. These data suggest the need for strategies to reduce the inevitable cryodamage to human sperm, and consequently improve the outcomes of sperm cryopreservation.

Myo-inositol (Myo-Ins) is the most biologically active form of inositol, a physiological compound belonging to the sugar family that is synthesized from glucose-6-phosphate in the human testis [11]. The concentration of Myo-Ins differs throughout the reproductive system, increasing along the epididymis and the vas deferens [12]. MyoIns acts as a second messenger in the cellular signal transduction pathway involved in the regulation of intracellular calcium $\left(\mathrm{Ca}^{2+}\right)$ concentration [13]. Myo-Ins, present in cell membranes, is involved in cell growth, lipid synthesis, cell cytogenesis, and morphogenesis [14].

Previous studies found Myo-Ins to play a positive role in the regulation of seminal plasma osmolarity and volume [15], the expression of proteins essential for sperm chemotaxis [16], and sperm capacitation and the acrosome reaction [17]. These data provoked interest in investigating the potential positive role of Myo-Ins in the treatment of male infertility through either oral administration or in vitro supplementation. Furthermore, incubation of semen samples with Myo-Ins resulted in a significant increase in sperm motility and the number of spermatozoa retrieved after swim-up in both normozoospermic men and patients with abnormal sperm parameters [12].

In a recent study, the fertilization rate and embryo quality significantly increased in intracytoplasmic sperm injection cycles when oocytes were injected with spermatozoa prepared in media enriched with Myo-Ins $2 \mathrm{mg} / \mathrm{mL}$ [18]. Additionally, Myo-Ins proved to be well tolerated by spermatozoa in vitro, demonstrating that it can be efficiently and safely used as an antioxidant in laboratory practice and for preparation of semen samples in ART [14]. The objective of this study was to investigate the effects of in vitro Myo-Ins supplementation of ejaculated human sperm, prior to cryopreservation, on the cryo-survival rate (CSR).

\section{Methods}

\section{Ethical approval}

This study was approved by the Institutional Research and Ethical Committees of the Faculty of Medicine, Sohag University, Egypt. Written informed consent was obtained from all participants before enrollment.

\section{Study design}

The study was conducted in the clinical andrology laboratory at Ajyal IVF Center, Ajyal Hospital, Sohag, Egypt, between September 2016 and May 2017. The study included semen samples obtained from men attending the infertility clinic for a routine infertility workup. Semen samples with azoospermia, leukocytospermia, or a vol- ume less than $1 \mathrm{~mL}$ were excluded. Semen samples delivered by men with a history of intake of antioxidants in the last 3 months prior to enrollment in the study were also excluded.

\section{Pre-freeze semen analysis}

Semen samples were collected by masturbation into a sterile container following 48 to 72 hours of sexual abstinence. Routine semen analysis was performed according to the latest (fifth) edition of the World Health Organization (WHO) guidelines, and the following parameters were evaluated: semen volume, sperm concentration $\left(\times 10^{6} / \mathrm{mL}\right)$, percent of total motility $(\mathrm{TM})$, percent of progressive motility (PM), percent of normal sperm morphology, and the seminal leukocyte count $\left(\times 10^{6} / \mathrm{mL}\right)[19]$.

\section{Preparation of Myo-Ins stock solution}

Myo-Ins (cell culture tested) was supplied in a powder form (product no. 17508; Sigma Aldrich Chemical, St. Louis, MO, USA). The MyoIns was weighed under completely aseptic conditions using a digital scale with $0.01 \mathrm{~g}$ precision, and $500 \mathrm{mg}$ of Myo-Ins powder was dissolved in $5 \mathrm{~mL}$ of a sperm washing and preparation medium (AllGrad wash; LifeGlobal, Guelph, ON, Canada) to prepare a Myo-Ins solution. The solution was sterilized using a bacteriological Nalgene $(0.45 \mu \mathrm{m})$ filter (Thermo Fisher Scientific, Waltham, MA, USA). Aliquots of $10 \mu \mathrm{L}$ of stock solutions were prepared, each containing $1 \mathrm{mg}$ of Myo-Ins, for in vitro supplementation of the first halves of the split semen samples before cryopreservation. Equal aliquots $(10 \mu \mathrm{L})$, only containing the sperm washing and preparation medium, were prepared for subsequent addition to the second halves of the semen samples (the control group). The prepared solutions were stored in the refrigerator between $2^{\circ} \mathrm{C}$ and $8^{\circ} \mathrm{C}$, and kept away from direct light sources.

\section{Pre-freeze Myo-Ins supplementation of sperm}

Following routine semen analysis, each semen sample was split into two equal aliquots ( $0.5 \mathrm{~mL}$ each). One aliquot ( $0.5 \mathrm{~mL}$ of semen) was supplemented with $0.5 \mathrm{~mL}$ of sperm freezing medium (LifeGlobal) plus a dose of $10 \mu \mathrm{L} / \mathrm{mL}$ of Myo-Ins, corresponding to $1 \mathrm{mg} / \mathrm{mL}$ (Myo-Ins/semen), according to the composition of the stock solution. The second (control) aliquot $(0.5 \mathrm{~mL}$ of semen) was supplemented with $0.5 \mathrm{~mL}$ of sperm freezing medium plus $10 \mu \mathrm{L} / \mathrm{mL}$ of sperm washing and preparation medium. Both aliquots were incubated for 20 minutes [18] prior to cryopreservation.

\section{Sperm cryopreservation}

Sperm cryopreservation was performed using the slow freezing technique. The sperm freezing medium (LifeGlobal) was brought to room temperature at least 1 hour prior to cryopreservation. It was added dropwise to each aliquot ensuring through mixing after each 
drop, reaching a final dilution of 1:1 to avoid osmotic stress [20]. Each aliquot was then loaded into a straw labeled with the patient's name and aliquot type (CBS High Security sperm straw; Cryo Bio System, Grenoble, France). The straws were suspended in liquid nitrogen vapor ( $10 \mathrm{~cm}$ above the level of the liquid nitrogen) at $-80^{\circ} \mathrm{C}$ for 15 minutes, before being stored in liquid nitrogen $\left(-196^{\circ} \mathrm{C}\right)$ for 1 week.

\section{Assessment of post-thaw sperm recovery}

The frozen samples were thawed at $37^{\circ} \mathrm{C}$, and the post-thaw percentages of TM, PM, and CSR were determined. The sperm CSR was calculated according to the following equation: $\mathrm{CSR}=$ post-thaw TM (\%)/pre-freeze TM (\%) × 100 [21].

\section{Statistical analysis}

The data were analyzed using IBM SPSS ver. 24.0 (IBM Corp., Armonk, NY, USA). The results of the pre-freeze and post-thaw semen analysis are presented as median and interquartile range (IQR; 25th and 75th percentiles). Friedman repeated-measure one-way analysis of variance was used for comparisons of the sperm concentration, percent of TM, and percent of PM among three matched groups (prefreeze, post-thaw Myo-Ins, and post-thaw control). The Wilcoxon signed-rank test was used to compare the CSR between the two matched groups (post-thaw Myo-Ins vs. post-thaw control). Hypothesis testing was two-tailed and $p$-values $<0.05$ were considered to indicate statistical significance.

\section{Results}

A total of 50 semen samples were collected from 50 men during routine infertility evaluations. Nine semen samples were excluded due to a history of recent administration of antioxidants $(n=3)$, azoospermia $(n=4)$, or leukocytospermia $(n=2)$. The median and IQR of age for the remaining participants $(n=41)$ was 36 years $(31-38$ years). Of the 41 semen samples included in the analysis, 15 met the WHO 2010 normal reference values for semen parameters [19], and accordingly, were categorized as normal semen samples, while 26 samples had one or more parameters below the lower reference limits, and were categorized as abnormal semen samples.

The median and IQR of age for the men with normal semen parameters (35.5 years [31-36]) was not significantly different from that of the men with abnormal semen parameters (36 years [31-39], $p=0.59$ ). The dilution of the semen aliquots using sperm freezing medium (1:1) prior to freezing resulted in a significant reduction of the postthaw sperm concentration in the Myo-Ins group $\left(25 \times 10^{6} / \mathrm{mL}[20\right.$ 40]) and the control group $\left(25 \times 10^{6} / \mathrm{mL}[20-30]\right)$ compared to the pre-freeze values $\left(50 \times 10^{6} / \mathrm{mL}[30-80] ; p<0.001\right.$ and $p<0.001$, respectively). The median and IQR of normal sperm morphology in the pre-freeze samples (5\% [3\%-5\%]) was not statistically significantly different from that of the post-thaw samples in the Myo-Ins group (5\% [3\%-5\%], $p=0.47)$ and the control group (5\% [3\%-5\%], $p=$ 0.15).

Comparisons of the pre-freeze and post-thaw sperm parameters in

Table 1. Comparison of pre-freeze and post-thaw sperm parameters in all semen samples with and without Myo-Ins supplementation ( $\mathrm{n}=41$ )

\begin{tabular}{lccccccc}
\hline \multirow{2}{*}{ Parameter } & \multirow{2}{*}{ Pre-freeze } & \multicolumn{2}{c}{ Post-thaw } & & \multicolumn{3}{c}{$p$-value } \\
\cline { 3 - 4 } & & Myo-Ins group & Control group & & P1 & P2 & P3 \\
\hline Total motility (\%) & $50(30-50)$ & $15(10-35)$ & $10(6-30)$ & & $<0.001$ & $<0.001$ & 0.15 \\
Progressive motility (\%) & $35(20-35)$ & $10(5-20)$ & $5(3-15)$ & & $<0.001$ & $<0.001$ & 0.14 \\
Cryo-survival rate (\%) & - & $40(25-70)$ & $30(13-58)$ & & - & - & 0.041 \\
\hline
\end{tabular}

Values are presented as median and interquartile range (25th and 75th percentiles). The $p$-values $<0.05$ were considered to indicate statistical significance. Myo-Ins, myo-inositol; P1, pre-freeze vs. post-thaw parameters in the Myo-Ins group; P2, pre-freeze vs. post-thaw parameters in the control group; P3, postthaw parameters in the Myo-Ins group vs. the control group.

Table 2. Comparison of pre-freeze and post-thaw sperm parameters in normal semen samples with and without Myo-Ins supplementation ( $\mathrm{n}=15$ )

\begin{tabular}{|c|c|c|c|c|c|c|}
\hline \multirow{2}{*}{ Parameter } & \multirow{2}{*}{ Pre-freeze } & \multicolumn{2}{|c|}{ Post-thaw } & \multicolumn{3}{|c|}{$p$-value } \\
\hline & & Myo-Ins group & Control group & P1 & $\mathrm{P} 2$ & P3 \\
\hline Concentration $\left(\times 10^{6} / \mathrm{mL}\right)$ & $50(40-90)$ & $28(24-40)$ & $25(25-31)$ & 0.001 & 0.001 & 0.62 \\
\hline Total motility (\%) & $50(50-52)$ & $35(30-45)$ & $30(20-40)$ & $<0.001$ & $<0.001$ & 0.24 \\
\hline Progressive motility (\%) & $35(35-36)$ & $23(14-31)$ & $20(10-27)$ & $<0.001$ & $<0.001$ & 0.25 \\
\hline Cryo-survival rate (\%) & - & $64(55-81)$ & $54(40-80)$ & - & - & 0.212 \\
\hline
\end{tabular}

Values are presented as median and interquartile range (25th and 75 th percentiles). The $p$-values $<0.05$ were considered to indicate statistical significance. Myo-Ins, myo-inositol; P1, pre-freeze vs. post-thaw parameters in the Myo-Ins group; P2, pre-freeze vs. post-thaw parameters in the control group; P3, postthaw parameters in the Myo-Ins group vs. the control group. 
Table 3. Comparison of pre-freeze and post-thaw sperm parameters in abnormal semen samples with and without Myo-Ins supplementation $(n=26)$

\begin{tabular}{|c|c|c|c|c|c|c|}
\hline \multirow{2}{*}{ Parameter } & \multirow{2}{*}{ Pre-freeze } & \multicolumn{2}{|c|}{ Post-thaw } & \multicolumn{3}{|c|}{$p$-value } \\
\hline & & Myo-Ins group & Control group & P1 & P2 & P3 \\
\hline Concentration $\left(\times 10^{6} / \mathrm{mL}\right)$ & $50(30-60)$ & $20(10-40)$ & $20(10-30)$ & 0.004 & 0.002 & 0.35 \\
\hline Total motility (\%) & $40(30-50)$ & $10(6-15)$ & $10(3-10)$ & $<0.001$ & $<0.001$ & 0.15 \\
\hline Progressive motility (\%) & $25(10-35)$ & $5(3-10)$ & $5(1-5)$ & $<0.001$ & $<0.001$ & 0.11 \\
\hline Cryo-survival rate (\%) & - & $38(20-50)$ & $23(12-30)$ & - & - & 0.031 \\
\hline
\end{tabular}

Values are presented as median and interquartile range (25th and 75th percentiles). The $p$-values $<0.05$ were considered to indicate statistical significance. Myo-Ins, myo-inositol; P1, pre-freeze vs. post-thaw parameters in the Myo-Ins group; P2, pre-freeze vs. post-thaw parameters in the control group; P3, postthaw parameters in the Myo-Ins group vs. the control group.

all semen samples $(n=41)$ with and without Myo-Ins supplementation are shown in Table 1. Comparisons of the pre-freeze and postthaw sperm concentrations and percentages of TM, PM, and CSR in the groups with normal semen $(n=15)$ and abnormal semen $(n=26)$ are shown in Tables 2 and 3, respectively.

\section{Discussion}

Despite advances in sperm cryopreservation over the last few decades, the process of sperm freezing and thawing is associated with variable degrees of sperm cryodamage, resulting in reduced postthaw sperm recovery. The viability of spermatozoa after cryopreservation usually does not exceed 50\% [22]. Many agents have been investigated as cryo-protectant supplements to ameliorate the hazardous effects of human sperm cryopreservation and improve postthaw sperm quality, including antioxidants such as vitamin E [23], glutathione [24], catalase [25], ascorbic acid-2-glucoside [26], and melatonin [27].

In this study, the cryopreservation of ejaculated sperm from infertile men resulted in a significant reduction of post-thaw TM and PM, whether semen samples were supplemented with Myo-Ins or not. The deleterious effect of cryopreservation on sperm motility has been consistently reported in previous studies $[7,10,28]$. The reduction of post-thaw sperm motility was attributed to cryo-induced mitochondrial damage, and the subsequent decrease of the intracellular concentration of adenosine triphosphate (ATP) [10]. Reduction of post-thaw sperm motility has also been correlated with increased reactive oxygen species (ROS) production [29], and decreased antioxidant defenses in cryopreserved semen [30]. In ROS-producing semen samples, the post-thaw recovery of sperm motility and vitality was significantly lower than in ROS-free samples [28].

In this study, the post-thaw TM and PM in the group of semen samples incubated with Myo-Ins, prior to cryopreservation, were higher than in the control group, although the difference was not statistically significant. The lack of statistical significance in the post-thaw TM and PM in the two treatment groups may be attributed to the wide range of variability of results of sperm motility among the study population and the relatively small sample size. Interestingly, the sperm CSR in the semen samples incubated with Myo-Ins prior to cryopreservation was significantly higher than that in the samples cryopreserved without Myo-Ins supplementation. A recent review highlighted the major role of TM, PM, and total motile sperm count (TMSC) in the assessment of semen quality after cryopreservation [31]. However, determining the sperm CSR may be a more accurate measure of post-thaw sperm recovery than TM, PM, or TMSC alone.

In a previous study, Myo-Ins supplementation of bovine semen, prior to sperm cryopreservation, resulted in a significant increase in TM and motion kinetics as determined by computer-assisted semen analysis [32]. A recent study coincubated fresh and thawed human semen samples with Myo-Ins for 15 minutes, and found a significant increase in TM and PM in the fresh samples and a significant increase in PM in the thawed samples [14]. Therefore, it can be speculated that in vitro Myo-Ins supplementation of semen samples may play a role in reducing the level of sperm cryo-damage, thereby enhancing post-thaw sperm recovery.

Further analysis of the data in this study indicated that a significantly higher CSR in semen samples supplemented with Myo-Ins was observed only in abnormal semen samples, not in normal semen samples. This finding is consistent with the report of a previous study that found no effects of Myo-Ins on mitochondrial function in spermatozoa from men with normal standard semen parameters [12]. Such findings may have important implications on the utility of Myo-Ins for cryopreservation of poor-quality semen samples.

The exact mechanisms by which Myo-Ins exerts its protective effects against cryo-damage are not clear and warrant further research. Sperm cryo-protection provided by Myo-Ins supplementation may be related, at least in part, to the antioxidant properties of Myo-Ins, which counteracts the oxidative stress-induced damage caused by cryopreservation [33]. The positive protective role of Myo-Ins may also be related to its role in the regulation of $\mathrm{Ca}^{2+}$ concentrations in 
the plasma membrane, mitochondria, acrosome, and neck regions of sperm [34].

Intracellular $\mathrm{Ca}^{2+}$ modulates the activity of several enzymes, including phospholipase $\mathrm{A}_{2}$, phospholipase $\mathrm{C}$, and protein kinase $\mathrm{C}$ [17]. An initial effect of $\mathrm{Ca}^{2+}$ is the activation of phospholipase $\mathrm{C}$, which hydrolyzes phosphatidylinositol to diacylglycerol and inositol 1,3,4-triphosphate. Activation of these intracellular mechanisms induce an increase of cytosolic $\mathrm{Ca}^{2+}$, and consequently an increase of mitochondrial $\mathrm{Ca}^{2+}$, which stimulates oxidative metabolism and ATP production [12].

Despite the interesting findings of this study, some limitations should be noted. First, the semen samples were not stratified according to the number or type of specific sperm abnormalities. Second, the infertile men were not categorized according to their clinical diagnoses; and third, a control group of men with proven fertility was not included. In conclusion, in vitro Myo-Ins supplementation of ejaculated human sperm from infertile men, prior to cryopreservation, resulted in a significant increase in the CSR. This observation was evident in semen samples with abnormal pre-freeze sperm parameters. Future studies are warranted to explore the effects of in vitro Myo-Ins supplementation on sperm DNA integrity and ART outcomes. Future studies are also required to examine the effects of Myo-Ins supplementation on the outcomes of cryopreservation of testicular sperm.

\section{Conflict of interest}

No potential conflict of interest relevant to this article was reported.

\section{References}

1. Rozati $H$, Handley T, Jayasena CN. Process and pitfalls of sperm cryopreservation. J Clin Med 2017;6:E89.

2. Agarwal A, Allamaneni SS. Disruption of spermatogenesis by the cancer disease process. J Natl Cancer Inst Monogr 2005;(34): 9-12.

3. Auger J, Sermondade N, Eustache F. Semen quality of 4480 young cancer and systemic disease patients: baseline data and clinical considerations. Basic Clin Androl 2016;26:3.

4. Zhu WJ, Liu XG. Cryodamage to plasma membrane integrity in head and tail regions of human sperm. Asian J Androl 2000;2:1358.

5. Hossain A, Osuamkpe C, Hossain S, Phelps JY. Spontaneously developed tail swellings (SDTS) influence the accuracy of the hypo-osmotic swelling test (HOS-test) in determining membrane integrity and viability of human spermatozoa. J Assist Reprod Genet 2010;27:83-6.

6. De Leeuw FE, Chen HC, Colenbrander B, Verkleij AJ. Cold-in- duced ultrastructural changes in bull and boar sperm plasma membranes. Cryobiology 1990;27:171-83.

7. Dejarkom S, Kunathikom S. Evaluation of cryo-injury of sperm chromatin according to computer controlled rate freezing method part 2. J Med Assoc Thai 2007;90:852-6.

8. Check ML, Check JH, Long R. Detrimental effects of cryopreservation on the structural and functional integrity of the sperm membrane. Arch Androl 1991;27:155-60.

9. Cross NL, Hanks SE. Effects of cryopreservation on human sperm acrosomes. Hum Reprod 1991;6:1279-83.

10. Donnelly ET, McClure N, Lewis SE. Cryopreservation of human semen and prepared sperm: effects on motility parameters and DNA integrity. Fertil Steril 2001;76:892-900.

11. Chauvin TR, Griswold MD. Characterization of the expression and regulation of genes necessary for myo-inositol biosynthesis and transport in the seminiferous epithelium. Biol Reprod 2004; 70:744-51.

12. Condorelli RA, La Vignera S, Bellanca S, Vicari E, Calogero AE. Myoinositol: does it improve sperm mitochondrial function and sperm motility? Urology 2012;79:1290-5.

13. Foskett JK. Inositol trisphosphate receptor Ca2+ release channels in neurological diseases. Pflugers Arch 2010;460:481-94.

14. Palmieri M, Papale P, Della Ragione A, Quaranta G, Russo G, Russo $S$. In vitro antioxidant treatment of semen samples in assisted reproductive technology: effects of myo-inositol on nemaspermic parameters. Int J Endocrinol 2016;2016:2839041.

15. Yeung $\mathrm{CH}$, Anapolski M, Setiawan I, Lang F, Cooper TG. Effects of putative epididymal osmolytes on sperm volume regulation of fertile and infertile c-ros transgenic mice. J Androl 2004;25:21623.

16. Bahat $A$, Eisenbach $M$. Human sperm thermotaxis is mediated by phospholipase $\mathrm{C}$ and inositol trisphosphate receptor $\mathrm{Ca} 2+$ channel. Biol Reprod 2010;82:606-16.

17. Dragileva $\mathrm{E}$, Rubinstein $\mathrm{S}$, Breitbart $\mathrm{H}$. Intracellular $\mathrm{Ca}(2+)$ $\mathrm{Mg}(2+)$-ATPase regulates calcium influx and acrosomal exocytosis in bull and ram spermatozoa. Biol Reprod 1999;61:1226-34.

18. Rubino P, Palini S, Chigioni S, Carlomagno G, Quagliariello A, De Stefani $S$, et al. Improving fertilization rate in ICSI cycles by adding myoinositol to the semen preparation procedures: a prospective, bicentric, randomized trial on sibling oocytes. J Assist Reprod Genet 2015;32:387-94.

19. World Health Organization. WHO laboratory manual for the examination and processing of human semen. 5th ed. Geneva: World Health Organization; 2010.

20. Sieme H, Oldenhof H, Wolkers WF. Mode of action of cryoprotectants for sperm preservation. Anim Reprod Sci 2016;169:2-5.

21. Varghese AC, Nandi P, Mahfouz R, Athayde KS, Agarwal A. Hu- 
man sperm cryopreservation. In: Rao KA, Agarwal A, Srinivas M, editors. Andrology laboratory manual. New Delhi: Jaypee Brothers; 2010. p. 196-208.

22. Watson PF. The causes of reduced fertility with cryopreserved semen. Anim Reprod Sci 2000;60-61:481-92.

23. Taylor K, Roberts P, Sanders K, Burton P. Effect of antioxidant supplementation of cryopreservation medium on post-thaw integrity of human spermatozoa. Reprod Biomed Online 2009;18: 184-9.

24. Gadea J, Molla M, Selles E, Marco MA, Garcia-Vazquez FA, Gardon JC. Reduced glutathione content in human sperm is decreased after cryopreservation: effect of the addition of reduced glutathione to the freezing and thawing extenders. Cryobiology 2011;62:40-6.

25. Moubasher AE, El Din AM, Ali ME, El-sherif WT, Gaber HD. Catalase improves motility, vitality and DNA integrity of cryopreserved human spermatozoa. Andrologia 2013;45:135-9.

26. Jenkins TG, Aston KI, Carrell DT. Supplementation of cryomedium with ascorbic acid-2-glucoside (AA2G) improves human sperm post-thaw motility. Fertil Steril 2011;95:2001-4.

27. Karimfar MH, Niazvand F, Haghani K, Ghafourian S, Shirazi R, Bakhtiyari $S$. The protective effects of melatonin against cryopreservation-induced oxidative stress in human sperm. Int J Immunopathol Pharmacol 2015;28:69-76.
28. Mazzilli F, Rossi T, Sabatini L, Pulcinelli FM, Rapone S, Dondero F, et al. Human sperm cryopreservation and reactive oxygen species (ROS) production. Acta Eur Fertil 1995;26:145-8.

29. Chatterjee S, Gagnon C. Production of reactive oxygen species by spermatozoa undergoing cooling, freezing, and thawing. Mol Reprod Dev 2001;59:451-8.

30. Bilodeau JF, Chatterjee S, Sirard MA, Gagnon C. Levels of antioxidant defenses are decreased in bovine spermatozoa after a cycle of freezing and thawing. Mol Reprod Dev 2000;55:282-8.

31. Palomar Rios A, Molina Botella I. Sperm parameters that play a major role in the assessment of semen quality after cryopreservation. J Assist Reprod Genet 2017;34:1271-6.

32. Bucak MN, Tuncer PB, Sariozkan S, Baspinar N, Taspinar M, Coyan $K$, et al. Effects of antioxidants on post-thawed bovine sperm and oxidative stress parameters: antioxidants protect DNA integrity against cryodamage. Cryobiology 2010;61:248-53.

33. Coballase-Urrutia E, Pedraza-Chaverri J, Camacho-Carranza R, Cardenas-Rodriguez N, Huerta-Gertrudis B, Medina-Campos $\mathrm{ON}$, et al. Antioxidant activity of Heterotheca inuloides extracts and of some of its metabolites. Toxicology 2010;276:41-8.

34. Ho HC, Suarez SS. Characterization of the intracellular calcium store at the base of the sperm flagellum that regulates hyperactivated motility. Biol Reprod 2003;68:1590-6. 